\section{Don't let furore over neutrinos blur results}

Neutrinos have been in the news again - and not just because of the debate over last year's OPERA experiment at the Gran Sasso National Laboratory in Italy, the results of which gave rise to the mistaken claim that the particles could travel faster than light (Nature 484, 287-288; 2012). In March, multinational experiments at the Daya Bay reactor in Guangdong Province, China, tracked down a fundamental parameter that describes neutrino oscillations, and Fermilab's MINERvA experiment near Chicago, Illinois, transmitted a message using neutrino communication for the first time.

In the wake of these exciting findings, neutrino physicists should not be too tempted to release new data ahead of thorough analysis and ratification, which could harm science. Neutrinos are elusive and neutrino experiments are extraordinarily complex, so physicists should remain sceptical and ensure that results are solid before they are proclaimed to the tax-paying public.

Tommy Ohlsson KTH Royal Institute of Technology, AlbaNova University Center, Stockholm, Sweden.tommy@theophys.kth.se

\section{Control electronic waste in India}

Legislation that came into effect in India this month aims to deal with the environmental effects of electronic waste in the country. According to a government report, this waste stream has increased by a factor of more than five in seven years and is expected to exceed 800,000 tonnes in 2012.

However, the new law does not ban the dumping of toxic electronic waste from overseas, which contributes a further 50,000 tonnes. This is in violation of the 1992 Basel Convention, which restricts disposal and transboundary movements of hazardous waste, particularly from developed to developing countries.

India should follow China's example and stand firm against the dumping of electronic waste by the European Union and the United States, for example. It must tighten up enforcement of the Indian Supreme Court's 1997 blanket ban on the import and export of hazardous waste, in line with the Basel Convention.

To tackle India's domestic electronic waste, the new law stipulates that manufacturers must follow strict collection and recycling procedures, including a buy-back system (see go.nature. com/48fdyn). It is essential that these measures are rapidly implemented and then properly enforced by the state pollutioncontrol boards.

Govindasamy Agoramoorthy Tajen University, Yanpu,

Pingtung, Taiwan.

agoram@mail.tajen.edu.tw Chiranjib Chakraborty Vellore Institute of Technology (VIT) University, Tamil Nadu, India.

\section{Preserve Brazil's aquatic biodiversity}

Brazil's aquatic biodiversity is under threat from a proposed law that aims to boost degraded fishery resources. If approved, the law - put forward by Nelson Meurer of the Brazilian National Congress - would allow the cultivation of non-native fish species in freshwater aquaculture cages, overriding the currently prohibited introduction of non-native species into Brazil.

The fish that would be introduced are tilapia and carp species, and other species that are potentially invasive in Brazil (J. R. S. Vitule et al. Fish Fish. 10, 98-108; 2009). If these were to escape, they would further disrupt native freshwater biodiversity, which is already compromised by dam construction and pollution.

Politicians should instead be creating mechanisms to preserve native fauna and ecosystem functions, helping to realize Brazil's potential as a model for biodiversity conservation in the spirit of next month's Rio+20 conference. Meeting socio-economic needs must have the backing of sound environmental research.

Jean R. S. Vitule* Federal University of Paraná, Curitiba, Paraná, Brazil.

biovitule@gmail.com

${ }^{*}$ On behalf of 5 co-authors (for a

full list, see go.nature.com/9yvvk1).

\section{In defence of the animal model}

Jocelyn Rice points out perceived shortcomings of the experimental autoimmune encephalomyelitis mouse in modelling multiple sclerosis and in advancing effective human treatments for this disease (Nature 484, S9; 2012; online only). However, her title ('Animal models: Not close enough') seems to cast doubt on the value of animal models in general for developing therapeutic strategies. Even if unintended, this implication undermines efforts to narrow the gap between research and the clinic.

We all share the author's frustration over the lag that separates direct medical benefits from animal research. But to shorten it, we should foster the possibilities of each model, not malign them.

Selective reporting of animal models that fail to deliver anticipated therapies risks promoting misperception among clinical researchers and policymakers. They are more likely to remember that one mouse model was "worryingly unreliable" for screening multiple sclerosis treatments than they are to recall that mice were crucial in the development of many life-saving therapies.

Examples include ipilimumab, an antibody therapy that extends life in patients with metastatic melanoma; losartan, which reduces aortic disease in patients with Marfan syndrome; and the retinoic acid/arsenic trioxide therapy that saves the lives of patients with acute promyelocytic leukaemia. Richard M. Baldarelli The Jackson Laboratory, Bar Harbor, Maine, USA.

richard.baldarelli@jax.org

\section{The social sciences are already relevant}

Luk Van Langenhove argues that the social sciences should be made more relevant (Nature 484, $442 ; 2012)$. But the problem is rather that society remains largely unaware of the thousands of social-science studies produced every year that are relevant to global challenges such as climate change. Efforts should focus on increasing the societal use of scientific knowledge instead of producing more, starting with better communication of research findings to the public.

Van Langenhove notes that just 1,600 papers out of all socialscience publications in 2010 (1.6\%) contain the keywords 'environmental change' or 'climate change'. But keywords are not always the best way to rate the impact of different topics. Articles that might be relevant do not always mention the right keywords, and findings depend on keyword choice (for example, when I included 'sustainability' in Van Langenhove's search, a further 1,493 papers appeared). Social-sciences papers still score more hits for these keywords than papers in the natural sciences $(0.65 \%)$.

Other topics relevant to society include cancer, AIDS and obesity, which I found to score 4,173, 3,341 and 2,365 social-science articles, respectively, for 2010 . The social sciences are therefore already contributing substantially to solving societal issues.

Frank J. van Rijnsoever Copernicus Institute of Sustainable Development, Utrecht University, the Netherlands.

f.j.vanrijnsoever@uu.nl 